\title{
Review Paper: Reviewing the Status of Professional Commitment Principles in the Physician-Patient Communication Models
}

\author{
Shahriar Mousavinejad ${ }^{1}$ (D, Mehrzad Kiani ${ }^{1}$, Shabnam Bazmi ${ }^{1^{*}}$ (D)
}

1. Department of Medical Ethics, School of Traditional Medicine, Shahid Beheshti University of Medical Sciences, Tehran, Iran.

$\begin{aligned} & \text { Use your device to scan } \\ & \text { and read the article online }\end{aligned}$
$\begin{aligned} & \text { Cittation: Mousavinejad Sh, Kiani M, Bazmi Sh. Reviewing the Status of Professional Commitment Principles in the Physi- } \\ & \text { cian-Patient Communication Models. International Journal of Medical Toxicology and Forensic Medicine. 2019; 9(4):181-190. } \\ & \text { https://doi.org/10.32598/ijmtfm.v9i4.26133 }\end{aligned}$
dol ${ }^{\circ}$ https://doi.org/10.32598/ijmtfm.v9i4.26133

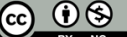

Article info:

Received: 19 May 2019

First Revision: 05 Jun 2019

Accepted: 23 Aug 2019

Published: 01 Oct 2019

\section{Keywords:}

Professionalism-

Communication models,

Patient

\begin{abstract}
Much attention is paid to the importance of the relationship between patients and physicians and the ethical issues that have been considered from different perspectives. Moreover, different models have been developed to establish such a relationship. In one of the most advanced methods, Emanuel EJ categorized 4 paternalistic -informative-interpretive models that have been agreed upon by many medical professionals. Each model has its characteristics and in the relationship between a patient and a practitioner, and to some extent, the professional commitment values (professionalism). In other words, the observance of the values of professionalism in each of these models has been based on the definitions of these principles; each is of particular importance, and individual attention has been paid to these values in different models. The present work aimed to evaluate each communication model according to the values of medical resource planning. Analyzing and adapting these models in terms of professional ethics could finally lead to analyzing the comparative approaches to professional commitment values.
\end{abstract}

\section{Introduction}

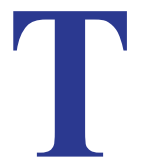

he social life of humans will almost be meaningless without communication. Relationships and their related skills are effective on the health and future of communication [1]. Communication indicates the transmission of messages between at least two people that can be direct, indirect, verbal, and non-verbal $[2,3]$. Several communication models are defined. For example, indirect or indirect communication, the relationship be- tween two people can be in the form of a brochure's information, educational picture, etc.; an indirect or direct relationship that can occur between the physician and the patient (a medical treatment system, and the medical staff, accordingly). This kind of communication, long known as a particular connection type, has always been the subject of attention by experts in different fields due to the particular conditions of patients and physicians.

An appropriate patient-clinician relationship importantly affects building confidence, helping with diagnosis, and improving the patient's health status $[4,5]$. In ancient

* Corresponding Author:

Shabnam Bazmi, MD.

Address: Department of Medical Ethics, School of Traditional Medicine, Shahid Beheshti University of Medical Sciences, Tehran, Iran

Tel: +98 (912) 2575330

E-mail:sh_bazmi2003@yahoo.com 
times, in the absence of paraclinical facilities, all of the diagnosis was based on the findings of talking to the patient and obtaining relevant information through such relationship; however, currently, most of the required data for diagnosis and treatment are obtained through the information provided by the physician for the patient. Hippocrates has also mentioned this point in $400 \mathrm{BC}$; he believes that creating a strong and effective relationship in risky situations is effective in the treatment [6]. Balint also expresses "not only medicine or pills, but also the way the physician gives them to the patient, and the whole condition where the drug is prescribed is important" $[6,7]$.

Appropriate communication with patients, in addition to being crucial for the treatment and their satisfaction, also has a significant role in reducing the medical staff complaints. Moreover, it is beneficial in developing a satisfaction sense in medical professionals.

The patient-physician relationship between has various aspects, including emotional and informational components. Furthermore, from Di Blasi's viewpoint, "emotional care" and "cognitive care" are referred to relying on this division [8]. Emotional care includes mutual trust, empathy, respect, truth, acceptance, and warmth; cognitive care includes data collection, sharing medical information, patient education, and the management of expectation [8].

By paying attention to the statements mentioned above, the importance of patient-physician relationship and its associated factors will be well understood. From another perspective, the patient-physician relationship is based on three primary roots. 1- Rooted in the mutual perception of social contracts and obligations. 2- Rooted in the development and transition from the stage of the traditional, historical relations of society up to now. 3- The personal origins arising from individuals' power in communicating, and considering special individual abilities [9]; specific models are considered for this purpose.

Other patient-physician communication kinds are defined based on experts' interests in communication. These types include physician-centered, patient-centered, and disease-centered models; their focus is on the role of each criterion and the centrality of communication in the basis of that factor as the communication axis. In Parsons's functional sick role model, the individual is considered as a part of the entire community, and considers the disease to be a transient period that impedes the full functioning of the individual. Parsons considers the relationship basis to focus on overall patients' performance, thier disorders, and attempts to improve their social and individual performances. In addition, the two-way partnership model presented by Hollender and Szasz considers the active participation of both communication parties, as the basis of concepts, such as human equality and the need for mutual understanding of this equation; they consider physicians at the same level as patients in terms of autonomy and decision-making.

The Veatch Council model has been evaluated based on consultation, equality and friendship between the physician and the patient. It is known as a conventional model. Another model considered more important interests than the patient's benefits, such as general health or important therapeutic or scientific goals. In this model, the individual's autonomy is totally ignored and can be considered as the exacerbated form of the patriarchal model; it also is recognized as the instrumental model in some sources.

\section{Emanuel's Proposed Model}

One of the most common communication models between physicians and treatment staff with the patient is based on Emanuel EJ studies. It introduces 4 main models ruling over the patient-physician relationship [10], including parental, informative, interpretative, and deliberative kinds. Given that most experts agree on this categorization, we considered this model as consistent with the objectives of this article and briefly overviewed of it.

\section{Parental Model}

In most world cultures and regions, from immemorial times, medicine had a remarkable fusion with wise people and religious missionaries and clerics; even in some cultures, witches and wizards, or physicians had a particular social class. Furthermore, the patient-physician relationship, due to its specific characteristics and the patient-physician differences in terms of information and recognition of the disease and the power to treat the disease have been specifically taken into account. In such relationship, the physician had a higher class than the patient, and due to having the information and ability to diagnose and treat diseases, had greater power than the patient. Moreover, the patient, due to the need for the physician's help and being in the position of demand was in a lower position than the physician.

This communication model is in an unequal condition in terms of the class and known as a parent-child relationship in the model presented by Eric Berne's theory of "transactional analysis." In this model "parent" refers to a person who takes the responsibility of the conditions and trend of the relationship due to his/her upright posi- 
tion and power (in terms of decision-making and power). Furthermore, the "child" refers to a person who, for specific reasons, including a feeling of weakness toward the "parent" or the need for his/her confirmation, or need to attend that relationship, psychologically, tries to stay in the state that the "parent" wants [11].

In the patriarchal model, the physician, who is in the parent's position, due to having more abilities than the patient, has more control over the relationship and can lead the relationship. Besides, the patient executes his/ her orders because of the lack of knowledge about the disease and obeys the physician's orders due to the need to stay in this relation. In this model, the autonomy respect of the patient is severely damaged and reduced, and the physician plans and directs all the treatment process based on his/her information and values. This communication model is based on the philosophical theory of accountability; the physician should make the best decisions for his/her patient according to his/her professional tasks. In this model, physician is responsible for almost all treatment processes, and determining the best action for the patient based on his/her best interests is formed according to the physician's values and decisions. This model suggests that as a parent only wants all the good for his child, the physician also does not want bad happenings for the patient; all of his/her actions will supply the best interests of his patient [12].

Furthermore, the patient also ultimately puts his or herself at the disposal of the physician through reliance on the physician and the belief that his physician will never want bad things for him/her, and leaves the decisionmaking process to the physician [13]. Such complete trust can more clearly be observed in the oral culture of many countries, especially in the cultures where medicine, wisdom, and spirituality have long been combined. For example, in the Iranian culture, this statement is heard very much when the patient refers to a physician: "First God, second you." In this situation, the patient believes that God desires nothing but good for him/her; thus, the person trusts the will of God and considers the physician as a person with power at a lower level than God. However, physician is considered far higher than himself and ignores his autonomy. This is also known as the parental-patriarchal-priestly model $[14,15]$.

\section{Informative Model}

In this model, the relationship is known as the consumer model, the scientific model or the engineering model. Furthermore, the physician is known as a person aware of the patient's information and the results of the tests and examinations; the physician's role is to provide the patient with information. In this model, the patient makes the final decision about the treatment and its method according to the information provided by the physician. In this model, the patient's autonomy is more respected than the patriarchal model.

Additionally, the patient's independent and autonomous decisions are entirely accepted. The problem with this method is that in many cases, the physician's role is reduced to a machine level, providing information, and after the patient's decision making, the physician plays an executor role of the patient's orders. Another drawback of this model is that, in many cases, the patient does not know the valid values for the correct decision-making process and merely decides based on information that cannot even analyze accurately in some cases. In this model, clinical information analysis for the patient's maximum benefits, which is one of the professional duties of the physician, is severely neglected; accordingly, the patient acts based on the values that are within his/her current knowledge of therapeutic interventions $[10,16,17]$.

\section{Interpretative Model}

This model is ahead of the informative model and known as the counselor model. The physician, in addition to providing accurate information to the patient, must try to determine the patients' values and help them better understand their current position. In this model, autonomy concern is beyond the previous model, which virtually empowers the patient to make the decision together with providing information and helping to recognize the patient's values. In this connection, the physician has a more active role than the previous model; however, he does not intervene in the patient's decisions with his/her values and sentiment and does not impose anything on him. However, interpreting the data and helping the patient better understand the facts and personal values, makes it possible to make a decision closer to the best of the patient's interests; ultimately, it is the patient who makes the final decision. In this model, the physician's role is very similar to the role of a consultant who advises someone seeking help according to the data and actual values. However, the extent of the physician's related education and ability remains unclear in this method $[10,17,18]$.

\section{Deliberative Model}

This model is also a step beyond the interpretative model and known as the "friendship" or "teacher" mod- 
el. This model of communication is very similar to the interpretative model and is essentially a completed form of it. In this model, all efforts of the physician and patient are to determine the patient's health-related values and select the best option in this respect. In this method, the patients should get prepared for an open and developing conversation to determine their status, and the physicians should prepare for providing accurate information, interpretation, and explanation, and persuade the patients to make the best decision based on their patient's values and interests.

In principle, according to what is desired in the process of obtaining informed consent, by examining the patient's capacity, respect for autonomy and the discretion of decisions, and by providing accurate data and empowering the patient, the physician ultimately provides a merciful counsel like a compassionate friend or teacher; even some times convinces him/her to make decisions based on best interests and without pressure. In this model, the patient's autonomy is highly respected, and the attempt to recognize his/her health-related values is an essential part of this model [10, 17, 19].

The important difference between this model and the interpretative model is the more active role of the physician in recognizing the patients' values based on their interests and empowering patients in terms of their health-related values; even in some conditions, affecting the decision-making process, without pressure.

As previously mentioned, like a friend or teacher, in addition to helping the friend or student to understand the values, abilities and decision-making conditions, the physician will be able to create an optimal orientation in decision-making process with more attention and dominance on the whole subject. Moreover, he/she will provide compassionate consultation without imposing beliefs and values to make a decision based on the patients' best interests. While in the interpretative model, the physician, as a neutral advisor, is helping to recognize the values and enhance the patient's ability to make decisions based on the patient's best interests; all that ultimately leads to decision-making based on the knowledge, values, and wishes of the patient with the help of the physician. Therefore, the physician's active and influential role is less than the deliberative model $[10,16,19]$.

These models of communication with their specific characteristics and peculiarities have a particular position concerning the professional medical values; in each one, some parts have been strengthened or less considered.

\section{Definition of Professional Ethics}

Initially, the concept of professional ethics was used to refer to the occupational ethics. Today, some professional developers of ethics use the term's first meaning to define it. There are various definitions of professional ethics and some of them are mentioned below:

A) Professional ethics is a branch of ethics that tries to answer the ethical issues of various professions.

B) Professional ethics evaluate ethical issues and questions, values, and fundamentals in a particular profession, and examine ethics in the professional environment.

C) Professional ethics is a branch of moral knowledge, i.e. involved with professional ethical requirements and issues and professional definition; this is a specific activity that leads to individual guidance to determine a specific situation with particular ethics [20].

One of the most comprehensive definitions of professional ethics has been defined as a set of rules that individuals should follow voluntarily and based on the call of conscience and the mettle in performing professional work, without an external obligation of a legal penalty in the event of a violation [21].

\section{Gharamaleki also defines professional ethics as follows}

"Professional ethics, as a branch of moral knowledge, evaluates moral responsibilities in a profession and its ethical issues, and in the definition of the profession, it is considered to be a certain activity that leads the person to a designated position along with specific ethics" [22].

These definitions generally address the issue of professional ethics; while in some science fields such, as medicine, attorneyship, and education and training, more specific meanings are considered for that [23]. It is referred to as professionalism, which in medicine has been an ancient and respectable concept [24]. It was mentioned in most of the oath letters [25], and literally, it stems from the word professio meaning a general proclamation and a commitment to the society [23].

In other words, professional people declare that they are ready to provide services to protect the interests of the community through their specific capabilities, and this public announcement is accepted by the community. Professionalism is not an intrinsic and inherent privilege; 
it is rather a privilege that society grants to a person in return to his/her commitments. In fact, maintaining and safeguarding this privilege is vital and entirely depends on fulfilling individual's obligations as well as respecting the rules of interprofessional self-regulation which are appointed by the individuals of that profession to achieve their common goal.

For the purpose of gaining and remaining within the profession, some features are considered who are referred to as the principles of professionalism. Therefore, individuals must be committed to them and try to promote these principles. A list of these principles are mentioned below:

\section{Altruism}

Altruism indicates full respect for the service recipient with a humanitarian sense and empathy; that can include the patient's interests, paying attention to the patient's' demands, spending time and having the patience of providing information to them, and trying to reduce their anxiety and pains, establishing an active communication with the patient, and the active interaction of the physician with the treatment team or professional groups, or to teach their skills and knowledge to others [23, 26-28].

\section{Duty \& responsibility}

One of the most important pillars of professionalism is accountability and responsibility. Professional, in addition to being well aware of their duties and responsibilities, must try hard to fully provide them (e.g. punctuality, accountability, building trust, coordinating with the treatment team and the supervisor during teamwork and properly responding to requests, awareness of professional rules and attempting to perform it, including detecting errors and conflicts of interest, and avoiding from professional misconduct, and drug and alcohol abuse) [23, 27-29].

\section{Honor and integrity}

Honesty and loyalty are other characteristics of professionalism. They are essential in terms of interacting with service recipients and professional members, and issues such as honest behavior (honesty, fairness, being frank, punctuality), observing professional behavior principles such as behavior or dress codes, honesty in assessing their abilities, a fair and honest effort to promote the job position, the lack of taking advantages of social and scientific status, and observing ethical principles in research studies [23, 27-29].

\section{Respect for others}

Respect for patients and service recipients and their entourages, and people within the profession, are of the basis of ethical and humanity standards and the other requirements of professional behavior. These behaviors can include full respect for the autonomy of individuals and maintaining human dignity of patients and their families whether in their presence or absence, introducing yourself to them, not using medical terms in conversation with the patient, protecting the privacy of patients and colleagues in occupational and educational positions, providing counseling to the family or the legal guardian of the patient, and tolerance and patience against problems in accordance with professional qualifications [23, 27-31].

\section{Career Excellence}

Given the profession definition and commitment of the professionals to declare assistance to the community, the committed professionals must be aware of the latest scientific achievements at all stages of service provision and strive to acquire knowledge and science. Therefore, some items can be considered as headlines for their career excellence, such as the careful review of feedback from treatment behaviors, the attempts to selfassessment whether scientifically or physically, seeking help from colleagues in the event of a scientific or proficiency need, optionally attending in retraining programs, and adaptability and compatibility with new conditions in the health field and its changes as headlines for career excellence [15, 23, 27-29].

\section{Commitment to social justice}

The subject of justice and the attitude and performance of professionals in the field of medical issues or health policy-making significantly impacts fulfilling their obligations against service recipients and the community and those within the profession. Furthermore, items like recognizing the public health policies, resource distribution and its allocation in the field of health and resource care, medicine on the basis of cost-effectiveness, and the recognition and assessment of the priority of actions and situations are among them $[23,31]$.

We have defined the professional principles and reviewed various relation types between the physician and the patient. Accordingly, we evaluated the influence and development of each professionalism principle, in each relationship type. 
All 4 mentioned models are professionally accepted and valuable; however, considering that each focuses on particular aspects of the professionalism principles of, we have reviewed those separately.

In this study, the professionalism values have been investigated in 6 areas of altruism, responsibility, honor, and Integrity, respect for others, and commitment to social justice; each one of the 4 models mentioned in the relationship between physician and patient has been evaluated based on these values.

\section{Discussion}

To simplify evaluating the issue using simple computational methods, each patient-physician communication method has been analyzed and rated and analyzed by weighting each professionalism principle (very high attention $=5$, high attention $=4$, average attention $=3$, low attention $=2$ and very low attention $=1$ ). Table 1 (according to the rating based on the mentioned definitions) gives a general overview of the status of 4 models presented in the relationship between physician and patient in terms of professionalism. We briefly described the most critical factors involved in this rating concerning the definition.

\section{Altruism}

In principle, altruism is paying attention and considering the patient's demands and preferences, and trying to create an effective and supportive relationship concerning the most important goals of the patient-physician relationship. In the patriarchal model, supporting the patient and creating a parental and supportive role are highlighted; however, considering the acceptance of all responsibility for treatment by the physician and making decisions based on the best of the patient's interests in accordance with the values of the physician, the preferences of the patient's interests, and attention to their wishes are less critical, compared to those of other models. Therefore, it is less advantageous than the other three models. However, this does not reflect neglecting the patient's values or paying more attention to personal interests by the physician; the objective is instead paying attention to the best interests of the patient according to the physician's values. In the informative model, the physician is considered similar to a machine providing information to the patients about their health condition.

Moreover, in this model, much less attention is paid to the humanity and altruism aspect in comparison with other models. In the interpretative model, although physician makes more effort to help the patient based on the altruism definition, in comparison to the informative model; concerns like spending much time to calm the patient or paying full attention to their wishes are not considered in their premium level. In the deliberative model, however, the physician is beside the patients and tries as hard as possible to help them recognize personal values and empower them to decide with awareness and the most accordance with their best interests. Eventually, it leads to creating a sense of tranquility and empathy in them, and paying attention to the altruism in this model is much more evident $[13,17,19]$.

\section{Duty and responsibility}

The other principle of professionalism is the accountability of responsibility. Reviewing the definitions provided for communication models revealed that in all 4 models, a special attention has been paid to this issue. In the paternal model, the physician is entirely responsible for the treatment. Furthermore, to be able to achieve the best results, the physician must obligate to the highest responsibility level against the patient and the treatment. However, in the informative model, although the information is provided on the basis of duty, the way of data provision seems more mechanical, and other professional tasks during data provision, such as paying attention to the patient's psychological state or values and preferences are less considered in this model.

Table 1. General overview of the status of relationship between physician and patient in terms of professionalism

\begin{tabular}{|c|c|c|c|c|c|c|c|}
\hline Model & Altruism & $\begin{array}{l}\text { Duty and Re- } \\
\text { sponsibility }\end{array}$ & $\begin{array}{l}\text { Honor and } \\
\text { Integrity }\end{array}$ & $\begin{array}{l}\text { Respect } \\
\text { for Oth- } \\
\text { ers }\end{array}$ & $\begin{array}{l}\text { Career Excel- } \\
\text { lence }\end{array}$ & $\begin{array}{c}\text { Commitment } \\
\text { to Social } \\
\text { Justice }\end{array}$ & Rank \\
\hline Parental & 2 & 5 & 4 & 3 & 5 & 5 & 24 \\
\hline Informative & 2 & 3 & 4 & 4 & 4 & 3 & 20 \\
\hline Interpretative & 3 & 4 & 5 & 5 & 4 & 4 & 24 \\
\hline Deliberative & 5 & 5 & 5 & 5 & 5 & 4 & 29 \\
\hline
\end{tabular}


In the deliberative model, according to the definitions, the physician is beside the patient with the utmost endeavor to provide the best solutions for his/her decisionmaking. In both models mentioned above, the responsibility and the physician's accountability sense emerge at the maximum level; therefore, are scored the highest, compared to the other two models $[17,19]$.

\section{Honor and integrity}

There is no doubt that honor and integrity in the patient-physician relationship should be at its highest level. One of the most important requirements in a professional relationship, from the perspective of professional ethics, is honesty and integrity; it has been emphasized as an inviolable principle since the beginning of medical history and in the first oath letters. However, in the patriarchal model, in some cases, the physician's decision to provide incomplete information to the patient to support the patients' interests (e.g. having benevolent goals for the patient), may prevent providing some information and documentation to the patient. In addition, given the personal values of the physician, accurate and complete information may not be provided to the patient. This is the only point in the paternal model that has reduced its rating compared to other models. Additionally, in the informative model, in circumstances where the physician is asked, he/she may only advise on the collected range of information, and refrain from other actions that are consistent with the justice and honesty about the patient's status concerning their values and preferences. However, in the interpretive and deliberative models, the physician is obligated to contact the patient with the highest honesty, fairness, and clarity Levels possible [12, 13, 17].

\section{Respect for others}

Respecting others as a value in the field of professionalism does not merely mean respect for the sick person and polite behavior; it also includes paying attention to desires and respecting the patient's tendencies and values. The respect for others is well observed in all models; however, given the definition of respect for others, and above all, paying attention to the patient's autonomy, desires, and preferences, and participating the patient in the treatment process are considered as well. Thus, in the paternal model, the least attention is paid to the autonomy of the patients and their desires and preferences, in comparison with other models. However, in this model, all efforts of the physician are based on the patient's benefits from the viewpoint of the physician $[16,32]$.
In the paternal model, considering the acceptance of the entire responsibility for the treatment process from beginning to the end, reduced patient's participation in the decision-making and incomplete and intelligible explanation for the patient in some circumstances, less attention is paid to the patient's autonomy; accordingly, the reduction of patient's participation in making decisions in this model leads to a lower score than the two interpretative and deliberative models (those models focus on patient's autonomy and empowerment for optimal decision making at its maximum level). Moreover, in the informative model, although decisions are made based on patient's values and desires, the lack of necessary counseling to empower the patient and presenting it only as a mediator reduces the score of this model, compared to the two later models $[13,16,19]$.

\section{Career excellence}

In the case of career excellence, which is an important part of the professionalism, the physician in the patriarchal model, due to the need to adequate information to put him or her at a higher or parental level, and to assume all responsibility for the treatment, requires professional excellence, increased capabilities, and awareness of updated treatments and adaptation to the changing conditions and modern methods of treatment. In the deliberative model, the physician should be able to appropriately respond to all patient's questions and requests, provide accurate answers and counseling for the patient; thus, the physician requires career excellence and updates in personal information.

In informative and interpretative models, the need for sufficient and up-to-date information in the field of treatment should be seriously considered to provide information to the patient. Moreover, the physician should have sufficient information, and in the interpretative model, in addition to the presentation of the information, physicians should inform the patient about the value of desires, which requires adequate and up-to-date knowledge; however, less attention and need to career excellence is observed in this model, compared to the other two models. Of course, this does not mean that there is no attention paid to the issue of career excellence in these two models, and it is merely evaluated in comparison to the patriarchal and advisory models $[12,19]$.

\section{Commitment to social justice}

Commitment to social justice is among the specific criteria of professionalism and can have a different definition in mind or action depending on the attitude of each 
individual or the impact of the society. Another critical point is that full implementation of justice is complicated and in most cases, is not feasible because, in addition to the general health and macro politics that affect the treatment process, the physician's opinions will intervene in this case. Some believe that commitment to social justice is less considered in the patriarchal model, given its definitions and the central role of the physician in decision-making, which is practically more based on personal opinions. Additionally, because the physician is responsible for the whole treatment and requires career excellence and information updates as much as possible, they should be aware of the issues related to justice, such as resource allocation, health and public policies, the general health status of the community, and so on. Therefore, this comprehensive view will increase the physician's attention to social justice, in comparison to the other models.

In the informative model, the physician is the only provider of information, and the patient decides based on personal documents and values for therapeutic actions, and always makes decisions inconsistent with the general principles of justice. In the interpretative and deliberative models, given that final decision-making is based on the patient's autonomy, desires, and values, and in many cases, individual tendencies and values can interfere with public health policies, such as the cost-effectiveness of treatments, allocation, and supply of resources, prioritizing resources and so on. The probability of maximal attention of the physician to social justice is lower than that of the paternal model. However, compared to the informative model, the physician has more power to justify the patient and create sensitivity in him/her to make a fair decision. Therefore, it seems that attention to justice in the patriarchal and informative models is at the highest and lowest levels, and deliberative and interpretative models also take a position between these two models $[12,19]$.

\section{Conclusion}

Considering the aforementioned issues and examining each professionalism principles and their compatibility with patient-physician relationship models, the deliberative model pays the most attention to the principles of professionalism; the ability to present these principles in this model is more than other models. Additionally, in the informative model, it is possible to execute the principles of professionalism at its lowest level.

Therefore, trying to provide the deliberative model and driving physicians and patients to understand and execute this model and teaching it at the educational lev- els in medicine and relative majors are necessary. In this regard, more attention to teaching communication skills in the educational curriculum of medical students and holding educational courses in the form of short-term workshops and courses for physicians at the level of general practitioners and specialists can help achieve these goals. Paying attention to virtual educational products in the form of multimedia and other items, that can teach communication skills should be considered.

\section{Ethical Considerations}

\section{Compliance with ethical guidelines}

This is a review articleso it doen't need ethical code and consent form.

\section{Funding}

This research did not receive any specific grant from funding agencies in the public, commercial, or not-forprofit sectors.

\section{Author's contributions}

Idea of project, supervision of the study, revision of original draft and correcting the final draft: Shabnam Bazmi; Revision of original draft and correcting the final draft: Mehrzad Kiani; Idea of this: Shahriar Moosavinejad.

\section{Conflict of interest}

There is no conflict of interest.

\section{References}

[1] Norbakhshmoghadam A. [Define the relationship and its types (Persian)] [Internet]. 2017 [Updated 2017 Jan 30]. Availible from: https://public-psychology.ir/1396/08/communication.

[2] Ciara R. The interplay between nonverbal and verbal interactionin synthetic worlds which supports verbal participationand production in a foreign language. 2012 [Updated 2012 Dec 13]. Availible from: https://tel.archives-ouvertes.fr/tel00762382v2Submitted on 13 Dec 2012.

[3] Karbasiyan G, [Communication (Persian)] [Internet] 2014 [Updaterd 2014 Nov 15]. Availible from: http://pajoohe.ir/ \%D8\%A7\%D8\%B1\%D8\%AA\%D8\%A8\%D8\%A7\%D8\%B7Communication_a-31715.

[4] Banidavoodi Sh, Hoseizadeh M, Maragh E. [Communication with patients in Ahvaz Hospitals from Patient and observer' perspectives (Persian)]. Educational Developement of Jundishapur. 2015; 7(1):65-71. 
[5] Shafati M, Zahedi MJ. [Physician-patient relationship: Ethnography of multiple patient visits at private offices of doctors (Persian)]. Journal of Qualitative Research in Health Sciences. 2013; 2(1):46-61.

[6] Di Blasi Z, Harkness E, Ernst E, Georgiou A, Kleijnen J. [Influence of context effects on health outcomes: A systematic review (Persian)]. The Lancet. 2001; 357(9258):757-62. [DOI:10.1016/S0140-6736(00)04169-6]

[7] Michael and Enid Balint. The Balint Society. 2018 [Updated 2018 Sep 26]. Availible from: https:/ / balint.co.uk/

[8] Kelley JM, Kraft-Todd G, Schapira L, Kossowsky J, Riess H. The influence of the patient-clinician relationship on healthcare outcomes: A systematic review and meta-analysis of randomized controlled trials. PLOS One. 2014; 9(4):e94207. [DOI:10.1371/journal.pone.0094207] [PMID] [PMCID]

[9] Loewy EH, Loewy RS. Patients, society, and healthcare professionals. In: Loewy EH, Loewy RS, editors. Textbook of healthcare ethics. Berlin: Springer; 2002.

[10] Ezekiel J. Emanuel, Linda L. Emanuel, four models of the physician-patient relationship. Journal of the American Medical Association. 1992; 267(16):2221-6. [DOI:10.1001/ jama.1992.03480160079038] [PMID]

[11] Joines V, Stewart I, Transactional analysis B [Dadgostar, Persian trans.] Tehran: Dayereh; 2012.

[12] Drolet BC, White Cl. Selective Paternalism. Chicago: AMA Journal of Ethics; 2012.

[13] Beauchamp TL, Childress JF. Principles of biomedical ethics. Oxford: Oxford University Press; 2012

[14] The Stanford Encyclopedia of Philosophy. Paternalism [Internet]. 2014 [Updated 2014 June 4]. Availble from: https:// plato.stanford.edu/archives/sum2014/entries/paternalism/

[15] Razzaghi MR, Afshar L. A conceptual model of physicianpatient relationships: A qualitative study. Journal of Medical Ethics and History of Medicine. 2016; 9:14. [PMID] [PMCID]

[16] Agarwal AK, Murinson BB. New dimensions in patientphysician interaction: Values, autonomy, and medical information in the patient-centered clinical encounter. Rambam Maimonides Medical Journal. 2012; 3(3):e0017. [DOI:10.5041/ RMMJ.10085] [PMID] [PMCID]

[17] Oprea L. An analytic review of the doctor-patient relationship (part II). Revista Romana de Bioetica. 2009; 7(3):125.

[18] Arafat Y, Kabir R, Andalib A. A narrative review on progression of doctor-patient relationship model in light of time and culture. International Journal of Perceptions in Public Health. 2017; 1(2):102-7.

[19] Wagne Rn. The role of autonomy in the physician-patien relationship. Johnson City: East Tennessee State University; 2015.

[20] Biabani Sh, Asadbeigi S. Surveying the impact of ethics and professional conduct of eployees on customer loyalty. International Journal of Innovative Research in Science. 2013; 2(11):6405-11.
[21] Amiri A, Hemati M, Mobini M , [Professional ethics necessary; for the organization (Persian)]. Ma'rifat-i Ākhlaqī. 2010; $1(4): 137-59$

[22] Gharamaleki AF. [Professional ethics (Persian)]. Qom: Majnoon; 2006.

[23] Nemati S, Saberi A, Heidarzadeh A. [Medical professionalism and its education to medical students (Persian)]. Research in Medical education. 2010; 2(1):54-61.

[24] Seif-Farshad M, Bazmi Sh, Amiri F, Fattahi F, Kiani M. Knowledge of medical professionalism in medical students and physicians at Shahid Beheshti University of Medical Sciences and affiliated hospitals-Iran. Medicine (Baltimore). 2016; 95(45):e5380. [DOI:10.1097/MD.0000000000005380] [PMID] [PMCID]

[25] Bazmi Sh. Teaching professional values in medicine. Journal of Medical Education. 2016; 15(2); 63-4.

[26] Iranian Association for Ethics in Science and Technology. Professionalism in medicine (Persian) [Internet]. 2014 [Updated 2014 March 20]. Available from: http://iranethics.ir/ printme.php?

[27] Kanter MH, Nguyen M, Klau MH, Spiegel NH, Ambrosini VL. What does professionalism mean to the physician? The Permanente Journal. 2013; 17(3):87-90. [DOI:10.7812/TPP/12120] [PMID] [PMCID]

[28] Omar HasanKasule, Medical professionalism and professional organizations. Journal of Taibah University Medical Sciences. 2013; 8(3):137-41. [DOI:10.1016/j.jtumed.2013.09.003]

[29] Johns Hopkins Medicine. Roles and responsibilities. Maryland: Johns Hopkins Medicine; 2012.

[30] Alni JR, Borhani F, Ebadi A, Bazmi S. Professional ethical competence for medical students: A qualitative study. Electronic Journal of General Medicine. 2018; 15(3):em18. [DOI:10.29333/ejgm/85497]

[31] ABIM foundation. The Physician Charter. New York: ABIM foundation; 1889 .

[32] Parker M, Dickenson D. The Cambridge medical ethics workbook: Case studies, commentaries and activities. Cambridge: Cambridge University Press; 2010. 
This Page Intentionally Left Blank 